\title{
Phase Structure of Lattice QCD at Finite Density with Dynamical Fermions
}

\author{
I.M. Barbour ${ }^{\mathrm{a}}$, J.B. Kogut ${ }^{\mathrm{b}}$ and S.E. Morrison ${ }^{a *}$

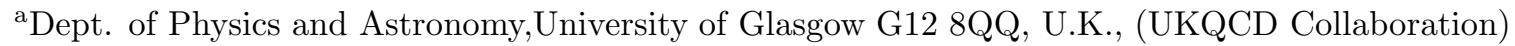 \\ ${ }^{b}$ Dept. of Physics,University of Illinois, 1110 West Green Street,Urbana, IL 61801
}

We compare the chemical potential associated with the onset of non-zero baryon number density on $6^{4}$ and $8^{4}$ lattices at $\beta=5.1$ and ma $=0.01$. We provide evidence for $Z(3)$ tunnelling. We determine a critical chemical potential of $\mu a \simeq 0.1$ which is unexpectedly low. We also determine the dependence of the onset of the observed phase transition on the quark mass. The physically misleading result of the quenched theory is shown to persist despite the inclusion of the complex fermion determinant.

\section{Introduction}

Lattice QCD at finite baryon density holds the key to an understanding of the phase transition between quark-confined hadronic matter and the quark-gluon plasma. At zero temperature we expect this transition to occur at $\mu=\frac{m_{p}}{3}$ corresponding to the lowest lying state with non-zero baryon number.

The fact that the fermion determinant becomes complex when $\mu$ is non-zero makes numerical simulations particularly difficult. Simulations using the quenched theory [1] give the physically misleading result that as the quark mass tends to zero, the lowest lying state with non-zero baryon number becomes massless. This corresponds to $\mu_{c} \simeq \frac{m_{\pi}}{2}$. Recent work by Stephanov [2] using the random matrix method has provided a solution to this particular problem by demonstrating that at nonzero $\mu$ the quenched theory is not a simple $n \rightarrow 0$ limit of a theory with $n$ quarks. This explanation implies that the phase of the determinant is important for a simulation of full QCD at finite $\mu$. However earlier work on the full theory at strong coupling [3] indicates that the transition still occurs at $\frac{m_{\pi}}{2}$.

\subsection{Formulation}

The GCPF can be expressed as an ensemble average of the fermionic determinant normalised

\footnotetext{
*talk presented by S.E. Morrison
}

with respect to the fermionic determinant at $\mu=$ 0 . This means that our probability weighting factor is well-defined.

$$
\mathcal{Z}=\frac{\int[d U]\left[d U^{\dagger}\right] \operatorname{det} M(\mu) e^{-S_{g}\left[U, U^{\dagger}\right]}}{\int[d U]\left[d U^{\dagger}\right] \operatorname{det} M(\mu=0) e^{-S_{g}\left[U, U^{\dagger}\right]}}
$$

which leads to:

$\mathcal{Z}=\left\langle\frac{\operatorname{det} M(\mu)}{\operatorname{det} M(\mu=0)}\right\rangle_{\mu=0}$

where $S_{g}$ is the pure gauge action and $M$ is the Kogut-Susskind fermion matrix:

$i M=G+i m+V e^{\mu}+V^{\dagger} e^{-\mu}$.

$G$ contains all the spacelike gauge links and the bare quark mass and $V$ all the forward timelike links. On each given configuration of the gauge fields $\operatorname{det} M$ is expanded as a polynomial in the fugacity variable with powers ranging between $\left[-3 n_{s}^{3}, 3 n_{s}^{3}\right] \quad\left(n_{s}\right.$ corresponding to the spatial extent of the lattice). The ensemble averages of the coefficients of this normalised expansion are the corresponding expansion coefficients for the GCPF.

The gauge field configurations are generated using Hybrid Monte Carlo, with the expansion coefficients of $\operatorname{det} M$ obtained from the eigenvalues of the propagator matrix $P$ (Gibbs 顿)

$P=\left(\begin{array}{cc}-G V & V \\ -V & 0\end{array}\right)$ 
Thus (for $a=1$ ):

$$
\begin{aligned}
& \operatorname{det}(i M)=e^{3 \mu n_{s}^{3} n_{t}} \operatorname{det}\left(P-e^{-\mu}\right) . \\
& \operatorname{det}\left(P-e^{-\mu}\right)=\sum_{n=0}^{6 n_{s}^{3} n_{t}} w_{n} e^{-n \mu}
\end{aligned}
$$

The eigenvalues, $\lambda$, of $P$ have a $Z\left(n_{t}\right)$ symmetry and a $\lambda$ and $1 / \lambda^{*}$ symmetry, which leads to an expansion of the form:

$$
\mathcal{Z}=\sum_{n=-3 n_{s}{ }^{3}}^{3 n_{s}{ }^{3}}<b_{|n|}>e^{n \mu n_{t}}=\sum_{n=-3 n_{s}{ }^{3}}^{3 n_{s}{ }^{3}} e^{-\left(\epsilon_{n}-n \mu\right) / T}
$$

\section{2. $Z(3)$ Tunnelling}

In the pure gauge theory we have $N_{c}$ equivalent vacua related by $Z\left(N_{c}\right)$ rotations. Tunnelling between the different $Z(3)$ vacua is much more probable in the confined sector than in the deconfined sector. Since the pure-gauge action as well as the integration measure are invariant under the $Z(3)$ transformation, the GCPF can also be written as:

$\mathcal{Z}(\mu)=\frac{\int[d U]\left[d U^{\dagger}\right] \operatorname{det} M\left(\mu+z_{3} / n_{t}\right) e^{-S_{g}\left[U, U^{\dagger}\right]}}{\int[d U]\left[d U^{\dagger}\right] \operatorname{det} M(\mu=0) e^{-S_{g}\left[U, U^{\dagger}\right]}}(8)$

Averaging over the three $Z(3)$ vacua would eliminate the triality non-zero coefficients and, since $Z(0)=1$, the sum of the triality zero coefficients should tend to 1 . As Fig. 1 shows, at $\beta=5.1$ and $m=0.01$, we obtained a clear signal for Z $(3)$ tunnelling. The sum of the triality zero coefficients shows a strong tendency to average to 1 whilst those of the triality one and two coefficients each tend to zero. This is consistent with simulating in the confined phase at $\mu=0$ as expected at this coupling and quark mass.

\subsection{Baryon Number Density}

We consider a description of the system in terms of the canonical partition functions for fixed particle number. This technique allows us to include only those triality zero coefficients which, at the end of the simulation, are positive and have a reasonable error. The chemical potential as a function of the baryon number density is obtained from the local derivative of the energy, $\epsilon_{n}$, of the state with $n$ fermions with respect to $n$.

$\mu(\rho)=\frac{1}{3 n_{s}^{3}} \frac{\partial \epsilon_{n}}{\partial \rho}$ where $\rho$ is the fermion density, $\frac{n}{3 n_{s}^{3}}$.

In Fig. 2 we show the number density, at $\beta=5.1, m=0.01$ on $6^{4}$ and $8^{4}$ lattices, as a function of chemical potential. Only the dependence at low densities is shown as it is only the coefficients $\left\langle b_{n}\right\rangle$ for $n$ small which are determined with reasonable statistical error. There is little difference between the $6^{4}$ and the $8^{4}$ number densities. The onset of non-zero baryon number density at $\mu_{c} \simeq 0.1$ is unexpectedly low.

\subsection{Mass Dependence of the Onset $\mu$}

Therefore we have also considered the chemical potential (the onset $\mu$ ) required to make the level with 3 quarks equally probable with the zero quark level. This ad-hoc definition takes account of the fact that the lowest coefficients are the most accurately determined and allows errors to be estimated directly. Fig. 3 shows the quark mass dependence of the onset $\mu$. Note that, from a study of the Lee-Yang zeros in the complex m-plane, we expect the system to undergo a 'deconfinement transition' in the region $m<0.01$ on a $6^{4}$ lattice at $\beta=5.1$. The scaling with $\sqrt{m}$ is clearly preferred, consistent with the onset of the transition being controlled, in part, by a Goldstone boson.

\section{Conclusions}

Lattice QCD at finite baryon density continues to exhibit a phase transition inconsistent with $\mu_{c} \simeq \frac{m_{p}}{3}$ despite the inclusion of the complex fermion determinant. It may well be that a successful formulation of QCD at finite density must override the effects of a Goldstone boson.

\section{Acknowledgements}

We would like to thank Misha Stephanov, Maria-Paola Lombardo and Ely Klepfish for very useful suggestions. This work was supported in part by the NSF through grant NSFPHY92-00148, by DOE, by PPARC through grant GR/K55554 and by NATO via grant CRG960002. Computations were performed on the C90s at NERSC and PSC. 


\section{REFERENCES}

1. I. Barbour,N. Behilil, E. Dagotto, F. Karsch, A.Moreo, M.Stone, H. Wyld, Nuclear Physics B275 (FS17)(1986) 296; J.B. Kogut, M.P. Lombardo and D.K. Sinclair, Phys. Rev. D51 (1995) 1282.

2. M.A. Stephanov, Phys. Rev. Lett. 76 (1996) 4472 .

3. I. Barbour, E. Klepfish, J.B. Kogut, M.P. Lombardo, S.E. Morrison, Work in Progress.

4. P. Gibbs Phys. Lett. B182 (1986) 369; I. Barbour and Z. Sabeur, Nucl. Phys. B342 (1990) 269.
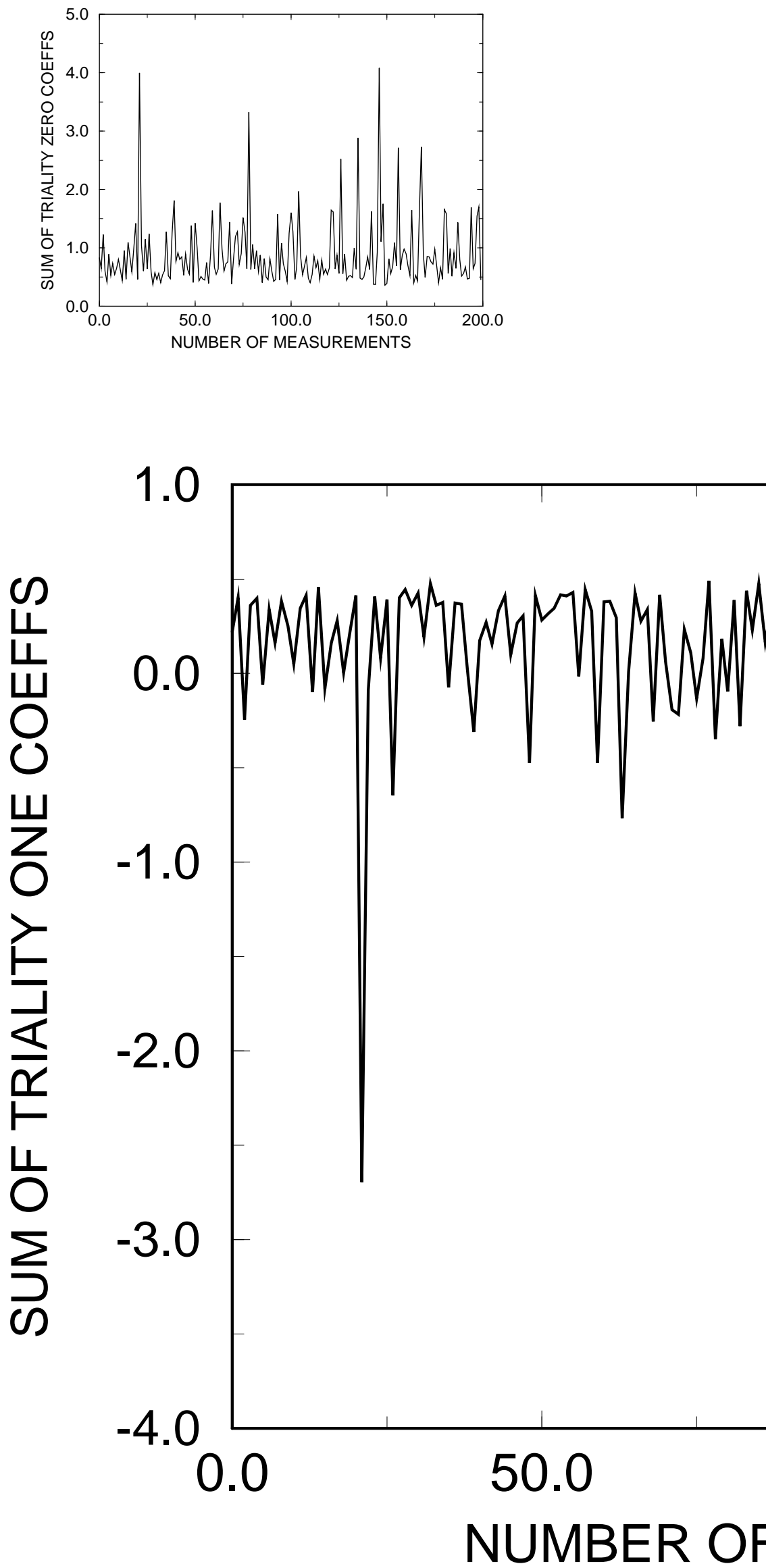

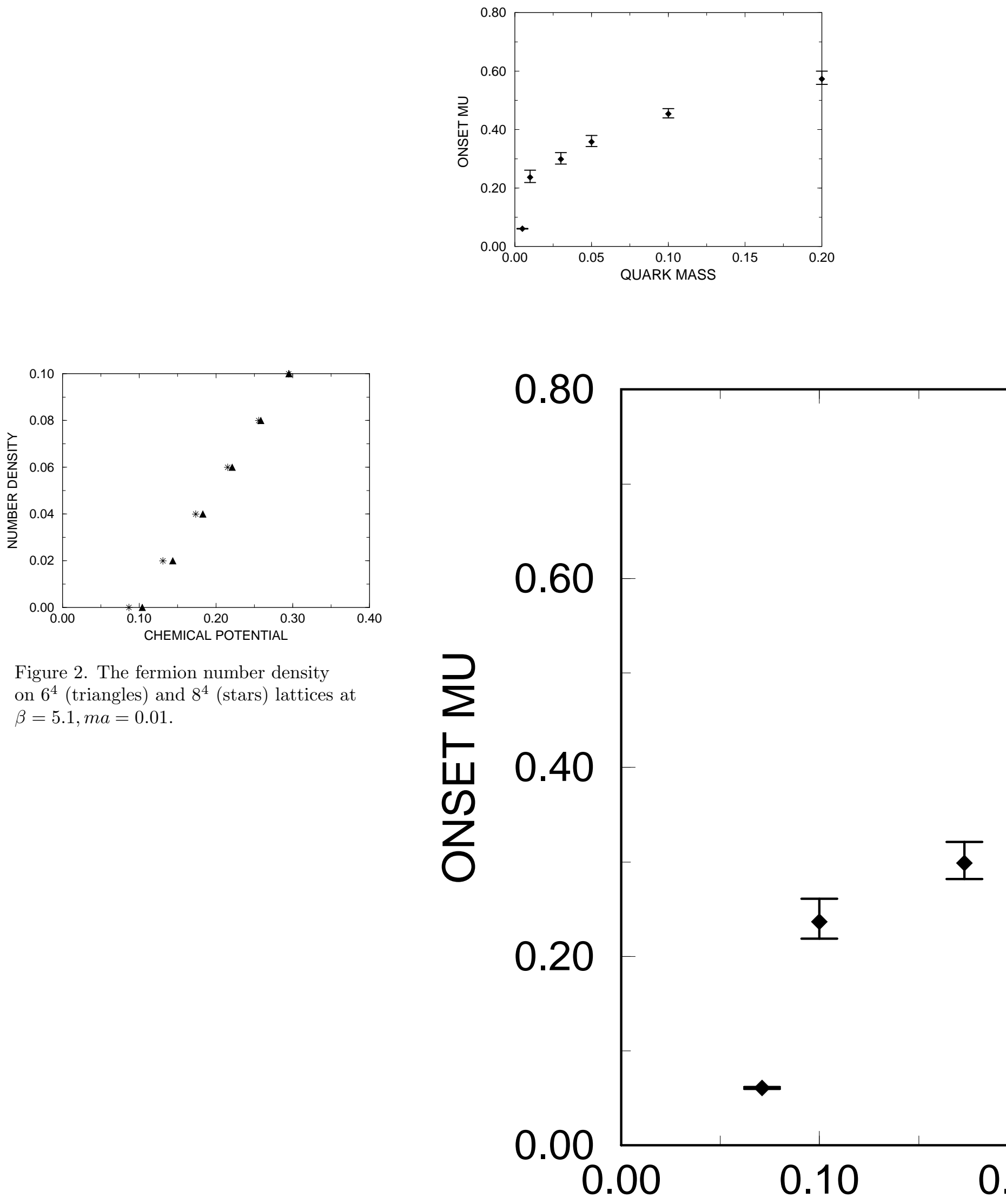

Figure 2. The fermion number density on $6^{4}$ (triangles) and $8^{4}$ (stars) lattices at $\beta=5.1, m a=0.01$. 